\title{
UN SISTEMA GENERAL DE LÓGICA NORMATIVA
}

\section{Introducción}

En los últimos años se ha despertado un gran interés por la sistematización de la lógica general de las normas y de los imperativos. Claro está que existe un parentesco muy íntimo entre normas e imperativos; nadie lo discute o ignora; mas cuál sea la naturaleza de tal parentesco, sí es motivo de disputa. Así, para los empiristas lógicos, la relación entre normas e imperativos es prácticamente de asimilación completa. ${ }^{1} \mathrm{Y}$ como ellos aducen que no hay una lógica de los imperativos en vista de que éstos no pueden -entre otras cosas- ser verdaderos o falsos, concluyen que no hay una lógica de las normas. ${ }^{2}$ Por otra parte, los filósofos alemanes y latinoamericanos que de una u otra manera continúan en la tradición kantiana, identifican también las normas con los imperativos; ${ }^{3}$ pero su opinión se extiende en un sentido contrario: ellos dan por sentado que existe una lógica general de las normas o imperativos. Los filósofos de esta confesión realmente no se interesan por la lógica general de las normas, sino por la "ontología del deber-ser"; ${ }^{4}$ dan más bien por sentado que de alguna manera la lógica general de las proposiciones descriptivas es también válida para las normas, a pesar de que se encuentran afirmaciones ocasionales en el sentido de que una lógica normativa sería distinta de la lógica ordinaria, aunque paralela a ella. ${ }^{5}$ Esta tesis se funda en la

1 Cf., por ejemplo, J. Jorgensen, "Imperatives and Logic", Erkenntnis (Viena) 7, 1938; A. J. Ayer, Language, Truth, and Logic, Victor Gollanez, Londres, 1936; cap. VI; Alf Ross, "Imperatives and Logic", Theoria (Upsala), 1941; E. L. Beardsley, "Imperative Sentences in Relation to Indicatives", Philosophical Review (New York), vol. LIII, 1944; C. L. Stevenson, Ethics and Language, Yale University Press, New Haven, 1945.

2 Para un examen de los dos argumentos principales en contra de una logica de imperativos cf. mi "A Note on Imperative Logic", Philosophical Studies (Minneapolis), $N^{\circ} 1$, vol. VI, 1955.

3 Como es bien sabido, Kant no hablaba de normas, sino de imperativos. Cf. Grundlegung, $2^{\text {a }}$ sección, al principio, donde define lo que es un imperativo.

4 Los ejemplos más notables son Max Scheler, Der Formalismus in der Ethik und die materielle Wertethik, Max Niemeyer, Halle, 1927; N. Hartmann, Ethics, vol. I, The Mácmillan Co., New York, 1932. Quizá la excepción más distinguida sea E. García Máynez, quien, aunque reconoce una ontología del deber-ser como básica, se ha interesado por sistematizar además la lógica normativa; en efecto, es justo reconocer que aunque García Máynez habla de "lógica jurídica", sus investigaciones son más generales, cubren toda la lógica normativa: Introducción a la lógica jurídica, Fondo de Cultura Económica, México, 1951; Los principios de la Ontología Formal del Derecho y su expresión simbólica, Imprenta Universitaria, México, 1953.

5 Cf. la sugestión de E. García Máynez en Introducción a la lógica jurídica, pág. 10: "Es importante percatarse de que no se trata de una aplicación, al campo del derecho, 
presuposición de que las normas no son verdaderas ni falsas, sino "válidas o inválidas": En ambos casos la lógica imperativa queda en el centro del problema de la lógica de las normas.

Entre tales posiciones extremas - una de extremo crítico y otra de extremo ingenuo- se encuentran otras posiciones. Pero casi todas se caracterizan porque han tratado de defender una lógica general de imperativos, salvaguardándose así la lógica de las normas como caso especial o como idéntica a ella; es decir, se trata de otra posición que de algún modo acepta también la tesis asimilativa de las normas a los imperativos. Ahora bien, los medios empleados para construir una lógica de imperativos varían grandemente. Unos lógicos meramente substituyen a los imperativos ciertas proposiciones indicativas. Así, por ejemplo, el imperativo "Juan debe ejecutar la

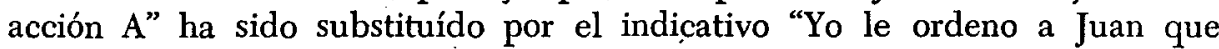
haga $\mathrm{A}^{\text {", }}$ " por el indicativo "Si C circunstancias están presentes, entonces está ordenado que Juan ejecute el acto A", 7 etc. Otros lógicos, por el contrario, han tenido la valentía de tratar de sistematizar directamente una lógica de imperativos. ${ }^{8}$ Finalmente, ha habido también esfuerzos por tratar de la lógica normativa independientemente de toda lógica imperativa, y se han obtenido resultados valiosísimos. ${ }^{9}$

Por mi parte, considero que el parentesco entre normas e imperativos es tan íntimo que una sistematización adecuada y de cierta complejidad tiene forzosamente que considerar tal parentesco. De otro lado, considero que en casi todos los intentos (por mí conocidos) de resolver el problema de la lógica normativa se ha partido de una presuposición falsa: la de que los imperativos tienen una logicidad paralela a la de los indicativos. ${ }^{10}$ Si se renuncia a la idea de una lógica plena de imperativos desaparecen algunos de los problemas suscitados por la crítica empirista. Esto es parte de lo que me propongo mostrar en este artículo, para lo cual tendré que examinar en gran

de las leyes supremas de la lógica pura. Mientras las últimas se refieren a juicios enunciativos, y afirman o niegan algo de su verdad o falsedad, los otros principios aluden a normas, y afirman o niegan algo de su validez o invaliảez. Aquéllas pertenecen, por ende, a la lógica del ser; éstos, a la del deber jurídico." (Subrayado del autor.)

6 Cf. Karl Menger, "A Logic of the Doubtful: On Optative and Imperative Logic" Reports of a Mathematical Colloquium, 2nd. series (Notre Dame University, Indiana), $\mathrm{N}^{\circ} 1,1939$; págs. 53-64.

7 Cf. Adrient Ledent, "Le statut logique des propositions impératives", Theoria (Upsala), vol. VIII, 1942; págs. 262-71.

8 El esfuerzo más valioso en este sentido es el de R. N. Hare, "Imperative Sentences", Mind (Edimburgo), n.s. vol. LVIII, 1949; y The Language of Morals, Clarendon Press, Oxford, 1952.

9 Ct. particularmente H. G. von Wright, "Deontic Logic", Mind (Edimburgo), n.s., vol. LX, 1951; y An Essay in Modal Logic, North-Holland Publishing Co., Amsterdam, 1951.

10 Esta insuficiencia de la lógica de los imperativos ha sido ya tomada en cuenta por A. Hofstadter y J. C. C. MacKinsey, "On the Logic of Imperatives", Philosophy of Science (Baltimore), vol. VI, 1939. 
detalle la relación que existe entre las normas y los imperativos, de una parte, $y$ entre las normas $y$ las proposiciones descriptivas $o$ indicativas orrdinarias, de otra. Alternativamente, puede decirse que en este estudio examinaré las relaciones fundamentales entre el ser y el deber-ser, en general y de una manera rigurosa, para llegar a resultados claros, precisos, sin ambigüedades. Me apresuro a indicar que, aunque lo que yo formulo en términos de lógica, hablando de proposiciones, de normas, de imperativos, de razonamiento, de reglas, de términos, puede muy bien traducirse a un lenguaje en que se hable de conceptos, de juicios, etc., así como en términos de ontologías, de deber-ser, de ser, de hechos, de realidades, etc., considero que la formulación lógica no sólo es más precisa, sino que es más exacta y literal. Realmente no puedo extenderme en este tema fundamental de la filosofía, que merece de por sí un amplio estudio. ${ }^{11}$ Baste ahora con que diga que un planteamiento del problema en términos de ser y deber-ser implica una asimilación de las normas o de los juicios con que pensamos el deber-ser a las proposiciones descriptivas o a los juicios con que pensamos el ser, ya que se corre el peligro de entender las normas como proposiciones en las que se predican de los objetos propiedades axiológicas en vez de propiedades meramente naturales. Más aún: hablar de ser y deber-ser en la forma en que corrientemente se hace es romper el Universo en dos zonas que después resulta imposible unir (salvo que se hable de un ser mayor, que cubre al deber-ser y al antiguo ser). Por otra parte, un planteamiento de la cuestión en términos de ser y deber-ser presupone una vez más la asimilación de las normas a los imperativos, y, por tanto, presupone que hay en los imperativos una logicidad plena.

\section{Normas e imperativos ordinarios}

Queda, pues, por lo menos sugerido que el planteamiento lógico es no sólo equivalente, sino preferible, al planteamiento ontológico. Esto es, lo que nos interesa es una clarificación sistemática de las relaciones lógicas entre normas, imperativos y proposiciones indicativas del tipo descriptivo. Esta clarificación se llevará a cabo mediante la exhibición de la estructura formal de todo sistema de normas de cierta complejidad; así, pues, no nos importará que las normas sean jurídicas, morales, éticas, usos sociales, reglas de juego, reglamentos, convenios, etc. Cualquier sistema de normas, de cualquier tipo o naturaleza, ha de conformarse a la estructura formal que aquí exhibiremos. Por eso hablamos de lógica general normativa.

Ahora bien: la exhibición de esa estructura formal se hará por medio de un sistema formal, en el cual aparecen esquemas o formas de normas, de imperativos y de proposiciones descriptivas, a efecto de no incluir ninguna in-

11 Cf. mi artículo "Naturaleza de los problemas filosóficos", Boletín Universitario, Universidad de San Carlos de Guatemala, $N^{*}$ 2, vol. IX, febrero de 1955. 
terpretación especial o concreta. De esa manera se logra mostrar la generalidad de las relaciones sistematizadas.

Como la lógica tiene siempre por objeto el estudio de la validez de los varios tipos de razonamiento, nuestra sistematización de la lógica de las normas ha de incluir la teoría de las deducciones más generales posibles dentro del campo de lo normativo. $Y$ por ello nuestro sistema formal ha de poseer una estructura deductiva; esto es, ha de proveer un conjunto de premisas siempre disponibles en todo razonamiento, por una parte, $y$, por otra, ha de suministrar los principios o reglas de inferencia deductiva a que se puede recurrir. Así, nuestro sistema tendrá irremisiblemente una forma axiomática - pues los axiomas son premisas siempre al alcance de la mano.

Con lo anterior se ha determinado ya la forma general del trabajo que nos toca realizar. Pero no hemos dicho nada acerca del contenido del sistema a construir. Por supuesto, queremos clarificar la estructura de lo normativo cualquiera que sea el tipo concreto de normas que lo "encarne", sean regulaciones para concursos, reglamentos de la revista Diánoia, normas jurídicas, reglas de ajedrez, damas chinas, futbol, etc. Así, pues, para garantizar la corrección de nuestro sistema formal nos conviene analizar, aunque sea en forma vaga e imprecisa, la naturaleza de los sistemas normativos ordinarios, ya que cada nota o característica que intuitivamente columbremos ha de quedar incorporada en el sistema, donde, además, tienen que encontrar una formulación precisa y una significación clara y exacta. He aquí algunas de esas características:

1. Normas, imperativos y proposiciones descriptivas ordinarias son tres tipos de oraciones irreductibles uno a otro.

2. Las oraciones imperativas no son ni verdaderas ni falsas, ni pueden servir como premisas o conclusión en ningún razonamiento.

3. Las normas, por el contrario, sí pueden servir como premisas y como conclusión en razonamientos; por ejemplo:

Si Carlos estudia, tú debes dejarlo solo.

Carlos estudia.

Por tanto: tú debes dejarlo solo.

El cajero debe cerrar la ventanilla a las cuatro y guardar el dinero.

Por tanto: El cajero debe guardar el dinero.

4. De otro lado, hay normas que valen o tienen aplicación o debemos obedecer, pero hay otras que no.

5. A cada oración normativa corresponde una oración indicativa ordinaria; por ejemplo:

"Todos deben decir la verdad" - "Todos dicen la verdad". 
"Juan debe decir la verdad" - "Juan dice la verdad".

"Si Miguel lo sabe, debe decirlo" - "Si Miguel lo sabe, lo dice".

6. Las normas y sus oraciones indicativas ordinarias correspondientes son mutuamente ininferibles, esto es, es inválido cualquier razonamiento que tiene a una de ellas por única premisa y a la otra por conclusión.

7. A cada norma corresponde un imperativo:

"Juan debe dormir" - "Juan, duerme".

"Todos deben decir la verdad" - "Digan la verdad".

"Si Miguel lo sabe, debe decirlo" - "Si lo sabes, dílo, Miguel".

8. En algún sentido, las normas incluyen o se asemejan a los imperativos.

9. En parte, esa semejanza consiste en que: (i) ni los imperativos ni las normas tienen por objeto describir el Universo, y (ii) ambos tipos de proposición tienen por objeto influir en el comportamiento o conducta de las personas.

10. Esta última característica en el caso de las normas es tal que la aceptación de "Yo debo hacer A" (esto es, la creencia sincera de que yo debo hacer A) me mueve a hacer A.

11. Las normas de algún modo indican que el correspondiente imperativo es vigente $o$ razonable o justificado.

Estas son apenas once de las características más notables de los imperativos y normas tal como aparecen en la lengua corriente. De ellas, nos interesarán muy particularmente las primeras ocho, por la sencilla razón de que son claramente formales. Nuestro sistema no puede, pues, dejar de incorporarlas y clarificarlas. La última es también formal, pero un poco más compleja, y la dejo para otra oportunidad. ${ }^{12}$ Las características 9 y 10 no son formales, pero una teoría filosófica completa ha de desarrollarlas con todo detalle; ${ }^{13}$ queden también para otra oportunidad.

\section{Paso hacia el sistema}

Antes de entrar a la construcción del sistema formal, que es justamente la formulación de mi teoría general de las normas, conviene hacer una presentación informal de la naturaleza del sistema, a efecto de: (1) hacer la teoría más comprensible, y (2) mostrar cómo las características arriba enumeradas se hallan en el sistema.

12 Este tema ha sido tratado en el capítulo 3 de mi The Logical Structure of Moral Reasoning, tesis doctoral presentada en abril de 1954 a la Universidad de Minnesota (inedita). Es parte, en el caso de las normas morales, de la teoría kantiana de la razón práctica.

${ }^{13}$ El sentido general (aunque un tanto confuso) que hay que darle al Achtung fürs Gesetzes de que habla Kant se encuentra, a mi juicio, en eso que llamé "aceptación" en el $N^{\circ}$ 10. Estudios muy valiosos han sido escritos por R. N. Hare, op. cit., y W. S. Sellars, "Some Reflections on Language Games", Philosophy of Science (Baltimore), vol. XXI, 1954; así como en correspondencia inédita entre ellos, que tuve el privilegio de conocer. 
Tomemos primero el concepto de verdad. En su aplicación a las proposiciones descriptivas tiene dos aspectos formales esencialísimos:

(a) Su intervención en la caracterización de la validez o corrección de una inferencia:

Un razonamiento es válido únicamente en el caso en que si se toma a las premisas como verdaderas es necesario tomar a la conclusión como verdadera.

(b) Su aspecto de correspondencia, de modo que si la proposición "Antonio está contento" es verdadera, entonces Antonio está contento.

Ahora bien, en las normas se pueden distinguir también las mismas propiedades. En el caso de los razonamientos se hace todavía más claro que las normas pueden ser verdaderas o falsas, ya que hay razonamientos mixtos, es decir, razonamientos en que entran premisas descriptivas y normativas, como sucede en uno de los ejemplos dados arriba. Si en estos casos se quisiera hablar de validez de las normas en contraste con la verdad de las proposiciones descriptivas, se encontrarían algunas dificultades o complicaciones en la caracterización de los razonamientos correctos. Véase que no hay nada anormal en decir: si es verdadera la norma "Carlos debe pagar su deuda", entonces Carlos debe pagar su deuda y viceversa.

Por tanto, diremos que las normas son verdaderas o falsas, y que las normas son también proposiciones, esto es, oraciones indicativas.

En cuanto a la diferenciación entre imperativos y proposiciones descriptivas, mi tesis se reduce a interpretarla como una diferenciación en el modo de combinarse los sujetos y los predicados, que son los mismos para ambos casos, según las características 5 y 7 , mencionadas en la sección II. Y convendremos en representar la combinación o predicación descriptiva por medio de paréntesis, y la combinación o predicación imperativa por medio de corchetes; por ejemplo:

"Luis busca a Juan" “Luis, busque a Juan"
Buscar (Luis, Juan) - Busear [Luis, Juan]

En cuanto a la diferenciación entre imperativos y normas (característica 1), que, a su vez, implica un parentesco íntimo (característica 8) entre ambos tipos de oraciones, a pesar de que las normas son también indicativas (resultado anterior), no encuentro más que una posibilidad: que la relación entre imperativos y normas sea constitutiva, no de inferencia. En otras palabras, cada norma lleva "dentro" un imperativo -el correspondiente (según característica $N^{0}$ 7). Así viene a explicarse en parte la comunidad de funciones del lenguaje imperativo y del lenguaje normativo (característica $\mathrm{N}^{\circ} 9$ ). Luego, la norma:

Felipe debe pagarle a Juan

contiene literalmente el imperativo: 
Felipe, páguele a Juan.

El problema se reduce ahora a entender cómo puede la oración anterior incluir a la última. Aquí lo que propongo es que se consideren la palabra "deber" (usada como verbo) y sus inflexiones como un término lógico, en el sentido de que si se aplica a un imperativo da por resultado un indicativo especial, tal como el vocablo "no" es un operador lógico que cuando se aplica a una proposición, digamos "Antonio estudia", da por resultado otra proposición, "Antonio no estudia". Claro está que el operador lógico "deber" es más complejo que el operador "no", pues se aplica a imperativos y da indicativos, entre otras cosas. En consecuencia, podríamos representar las oraciones correspondientes como sigue:

"Carlos da un libro a Juan" Dar (Carlos, libro, Juan). "Carlos, dé un libro a Juan" Dar [Carlos, libro, Juan]. "Carlos debe dar un libro a Juan" — Deber (Dar [Carlos, libro, Juan]).

Obsérvese que en la forma ilustrada con que simbolizamos los tres tipos de oraciones, se hace aparente la estructura lógica de cada una de ellas, y nos liberamos de los mecanismos gramaticales concretos y diferentes que las lenguas naturales utilizan. Puede verse fácilmente que las variedades gramaticales de tiempo, modo del verbo, construcción, preposiciones, declinación, etc., no afectan el hecho fundamental de que en una oración se habla de ciertos objetos y personas con cierto sentido, y este sentido queda plenamente expresado con el orden en que colocamos las palabras denotativas de los objetos y personas, de una parte, y con los paréntesis o corchetes, de otra. De la misma manera, una lengua puede no poseer palabra alguna que se traduzca por "deber" en castellano; pero si en esa lengua hay normas, habrá en ella un sufijo o prefijo, que afectando al verbo o al sujeto de la oración exprese un sentido normativo, o habrá en ella un tono o una entonación oracional, etc. Estas son cuestiones que los lingüistas estudian; a nosotros nos interesa sólo el concepto o sentido normativo, y como nuestro simbolismo lo destila o purifica, nuestro simbolismo resulta valioso, y nos evita confusiones. En efecto, introduciremos los símbolos usuales en la lógica moderna:

' -' para indicar negación;

$' v '$ para indicar disyunción;

' .' para indicar conjunción (esto es, el sentido de las conjunciones copulativas y adversativas ordinarias, y de los adverbios conjuntivos 'también', 'tampoco', etc.).

' $\rightarrow$ ' para indicar condicionalidad;

$' \equiv '$ para indicar bicondicionalidad.

Luego, las oraciones siguientes se simbolizan lógicamente como se expresa: 
"Si Luis viene, dale el libro" —_ Venir (Luis) $\rightarrow$ Dar [tú, libro, Luis].

"Carlos viene o no, pero tú debes darle el libro" -_ (Venir (Carlos) $v$-Venir (Carlos)). Deber (Dar [tú, libro, Carlos]).

\section{El sistema $N_{1}{ }^{\circ}$}

Después de esta breve exposición informal de los puntos dominantes de $\mathrm{mi}$ teoría general de las normas, paso a formularla con precisión y rigor. El sistema formal en que la exhibo será llamado el modelo o sistema $N_{1}{ }^{\circ}$, para distinguirlo de un modelo anterior al que denominé $N^{* 14}$.

1) Signos o expresiones primitivas (indefinibles) de $N_{1}{ }^{\circ}$ :

a) Nombres propios o constantes individuales: ' $a$, ' $b$ ', ' $c$ ',,.

b) "Pronombres" o variables individuales: ' $x$ ', ' $y$ ', ' $z^{\prime},{ }^{\prime} x_{1}$, ' ' $x_{2}$ ', ' $y_{1}^{\prime},{ }^{\prime} y_{2}{ }^{\prime}, \ldots$

c) Predicados o términos denotativos de propiedades (esto es, cualidades y relaciones): ' $A$ ', ' $B^{\prime},{ }^{\prime} C^{\prime}, \ldots$

d) Conectivos lógicos generales: '-' (que denota negación), ' $v$ ' (que denota alternación o disyunción), ' $O$ ' (que expresa disyunción mixta: imperativo-indicativa).

e) Operadores lógicos imperativos: ' $I$ ' (que sirve para referirnos al indicativo ordinario correspondiente a un imperativo o norma), ' $K$ ' (que equivale al uso de la palabra 'Deber' en la sección anterior).

f) Otros signos: '(',')', '[',']'.

Como todos estos signos son primitivos en $N_{1}{ }^{*}$, no hay definición posible de ellos en $N_{1}^{*}$; pero como su significado ha de quedar determinado de una manera precisa (además de las indicaciones generales y vagas dadas en la sección III), son necesarias las reglas que constituyen su significado, y que son de dos clases:

2) Reglas de formación de oraciones:

A) Las expresiones de las siguientes formas son indicativos y los únicos indicativos de $N_{1}{ }^{*}$ :

a) $Z(Y)$, donde $Z$ es un predicado de cualidad e $Y$ un nombre propio o una variable individual; $Z_{n}\left(Y_{1}, Y_{2}, \ldots, Y_{n}\right)$, donde $Z_{n}$ es un predicado de re-

$14 \mathrm{El}$ sistema $\mathrm{N}^{*}$ con una regla adicional de inferencia para imperativos, a lo cual llamé $\mathrm{C}^{*}$, se encuentra en mi An Essay in the Logic of Commands and Norms (inédita), tesis que para el grado de Master of Arts presenté a la Universidad de Minnesota en 1952. $\mathrm{N}_{1}{ }^{*}$ se diferencia de $\mathrm{N}^{*}$ en que tiene un axioma menos y en las restricciones impuestas a la regla de inferencia normativa; véase lo que sigue. 
lación de $n$ términos e $Y_{1}, Y_{2} \ldots, Y_{n}$ son constantes o variables individuales.

b) - $(Z)$, donde $Z$ es un indicativo.

c) $(X)(Z)$, donde $Z$ es un indicativo y $X$ es una variable individual.

d) $(Z) v(Y)$, donde $Z$ e $Y$ son indicativos.

e) $I(Z)$, donde $Z$ es un imperativo.

f) $K(Z)$, donde $Z$ es un imperativo.

g) $(Z) o(Y),(Y) o(Z)$, donde $Z$ es un imperativo de la forma $-(-(X) v(X))$ e $Y$ es un indicativo.

B) Las expresiones de las siguientes formas son los únicos imperativos de $N_{1}^{*}$ :

a) $Z[Y], Z_{n}\left[Y_{1}, Y_{2}, \ldots, Y_{n}\right]$, como en A) a).

b) $-(Z)$, donde $Z$ es un imperativo.

c) $(X)(Z)$, donde $X$ es una variable individual y $Z$ un imperativo.

d) (Z) $v(Y)$, donde $Z$ e $Y$ son ambos imperativos.

e) $(Z) o(Y),(Y) o(Z)$, donde $Y$ es un indicativo y $Z$ es un imperativo no equivalente a ningún otro imperativo de la forma $-(-(X) v(X))$.

Definiciones: Los otros signos usuales en lógica moderna se definen como de costumbre, excepto que ahora quedan generalizados para cubrir indicativos tanto como imperativos:

1. $(\mathrm{G} x(Z)=$ Def. $-(X)-(Z)$, donde $Z$ es un imperativo o un indicativo, y $X$ es una variable individual.

2. $(Z) \rightarrow(Y)=$ Def. $-(Z) v(Y)$, donde $Z$ e $Y$ son ambos indicativos o ambos imperativos.

3. $(Z) \rightarrow(Y)=$ Def. $-(Z) o(Y)$, donde $Z$ es un indicativo e $Y$ es un imperativo.

4. $(Z) \cdot(Y)=$ Def. $-(-(Z) v-(Y))$, donde $Z$ e $Y$ son ambos imperativos $o$ ambos indicativos.

5. $(Z) \&(Y)=$ Def.- $(-(Z) o-(Y))$, donde $Z$ es un indicativo e $Y$ es un imperativo.

6. $(Z) \equiv(Y)=$ Def. $((Z) \rightarrow(Y)) \cdot((Y) \rightarrow(Z))$, donde $Z$ e $Y$ son ambos indicativos o ambos imperativos.

3) Reglas de inferencia deductiva de $N_{1}{ }^{*}$ :

I. Modus ponens: Si dos indicativos de la forma $Z$ y $(Z) \rightarrow(Y)$ son demostrables en $N_{1}{ }^{*}$, entonces $Y$ también es demostrable en $N_{1}{ }^{*}$.

II. Regla de universalización: $\mathrm{Si}$ un indicativo $\mathrm{Z}$ es demostrable en 
$\mathrm{N}_{1}{ }^{\circ}$, entonces $(X)(Z)$ es también demostrable en $\mathrm{N}_{1}{ }^{*}$, donde $X$ es una variable individual.

III. Regla de inferencia normativa: $\mathrm{Si} Z$ e $Y$ son imperativos de $\mathrm{N}_{1}{ }^{\circ}$ $y$ el indicativo $(I(Z)) \rightarrow(I(Y))$ es demostrable en $N_{1}{ }^{\circ}$ sin necesidad de que se use ninguna de las siguientes proposiciones:

$$
\begin{array}{llrl}
(I(Z \vee I)) & \rightarrow(I(Z \circ I(Y))), & (I(Z \circ I(Y))) \rightarrow(I(Z \vee Y)), \\
(I(Z \vee I)) \rightarrow(I(I(Z) \circ Y)), & (I(I(Z) \circ Y)) \rightarrow(I(Z \vee Y)),
\end{array}
$$

$(\mathrm{I}(\mathrm{Z} \circ I(Y)) \rightarrow(I(I(Z) \circ \mathrm{Y})), \quad(I(I(Z) \circ Y)) \rightarrow(I(Z \circ I(Y)))$, entonces, el indicativo (normativo) $(K(Z)) \rightarrow(K(Y))$ también es demostrable en $N_{1}{ }^{*}$.

Las siguientes deducciones ilustran estos principios de inferencia (dando por sentado que las premisas son demostrables):

Regla I: Si Carlos viene, Arturo lo ve.

Carlos viene.

Por tanto: Arturo lo ve.

Regla II: Para cada hombre arbitrariamente tomado se cumple que ese hombre es mortal.

Por tanto: Todos los hombres son mortales.

Regla III: $\quad$ Si Carlos viene y mira a Arturo, entonces Carlos mira a Arturo.

Por tanto: $\mathrm{Si}$ es obligatorio que Carlos venga y mire a Arturo, es obligatorio que Carlos mire a Arturo.

4) Axiomas de $N_{1}{ }^{*}$ :

Las proposiciones de las formas siguientes son demostrables en $\mathrm{N}_{1}{ }^{\circ}$ :

(1) Axiomas para la lógica general: La versión de Paul Bernays del conjunto de Principia Mathematica (de Whitehead y Russell): ${ }^{15}$.

Al. $\quad \mathrm{Z} v \mathrm{Z} \rightarrow \mathrm{Z}$

A2. $Z \rightarrow Z \cup Y$

En estos cuatro axiomas. $X, Y$ y $Z$

A3. $Z v Y \rightarrow Y \vee Z$ son todos indicativos.

A4. $(Y \rightarrow Z) \rightarrow(X \vee Y \rightarrow X \vee Z)$.

15 Cf. D. Hilbert y W. Ackermann, Principles of Mathematical Logic, Chelsea Publishing Co., New York, 1950; págs. 27-8. 
(2) Axiomas para la lógica cuantificacional: Un conjunto que parece ser debido a F. F. Fitch: ${ }^{16}$

A5. $(X)(Y \rightarrow Z) \rightarrow((X)(Y) \rightarrow(X)(Z))$

A6. $\mathrm{Y} \rightarrow(X)(Y)$, siempre que la constante individual $X$ no sea libre en $Y$, esto es, o no ocurre en $Y$, o si ocurre en $Y$ ocurre en una parte $Z$ de $Y$, tal que $(X)(Z)$ es parte de $Y$.

A7. $(X)(Y) \rightarrow(Z)$, donde $Z$ es igual a $Y$ excepto en que todas las veces que la variable individual $X$ es libre en $Y$, la variable $T$ (no necesariamente distinta de $X$ ) es libre en $Z$, o $Z$ tiene un nombre propio.

En estos axiomas A5-A7 $Y$ y $Z$ son indicativos, $X$ y $T$ variables individuales.

(3) Axiomas para la lógica imperativa (o de los indicativos formados con ' 1 '):

$$
\begin{aligned}
\text { A8. } & I\left(Z_{n}\left[X_{1}, X_{2}, \ldots, X_{n}\right]\right) \equiv Z_{n}\left(X_{1}, X_{2} \ldots, X_{n}\right) \\
\text { A9. } & I(-Z) \equiv-I(Z) \\
\text { A10. } & I(Y \vee Z) \equiv I(Y) \vee I(Z) \\
\text { A11. } & I(Y \circ Z) \equiv Y v I(Z) \\
\text { A12. } & I(Z \circ Y) \equiv I(Z) \vee Y \\
\text { A13. } & I((X)(Z)) \equiv(X)(I(Z)) .
\end{aligned}
$$

En estos axiomas A8-A13 se supone que las expresiones son indicativos o imperativos, según las reglas de formación.

(4) Axiomas especiales para la lógica normativa (o de los indicativos formados con ' $K$ '):

A14. $K(Z) \rightarrow-K(-Z)$

A15. $K(Y . Z) \equiv K(Y) \cdot K(Z)$

A16. $K(Y \& Z) \equiv Y . K(Z)$

A17. $K(K(Z) \rightarrow Z)$

A18. $(X)(K(Z)) \rightarrow K((X)(Z))$.

En estos axiomas A14-A18 $Z$ e $Y$ son imperativos, menos en A16, don $Y$ es indicativo. $X$ es una variable individual.

En todos los axiomas Al-Al8 se ha hecho uso de las convenciones ordinarias sobre paréntesis: de modo que los signos lógicos tienen un radio de acción más reducido que el siguiente, en este orden: '-', ' $(X)^{\prime},{ }^{\prime}(\exists X)^{\prime},{ }^{\prime},{ }^{\prime}, v^{\prime},{ }^{\prime}{ }^{\prime}$, ' $\rightarrow$ ', ' $\rightarrow$ ', y $\cong$; además, se suprimen los paréntesis que separan o rodean a una sola oración.

16 Cf. W. V. O. Quine, Mathematical Logic, Harvard University Press, Cambridge, 1951; pág. 89. También A. Burks, "The Logic of Causal Propositions", Mind, n.s. vol. LX, 1951; pág. 379 nota. 
Por otra parte, valen las definiciones acostumbradas de prueba, demostración; variable libre y variable ligada.

Interpretación de los axiomas: El conjunto (1) de los axiomas A1-A4 establece ciertas propiedades formales de los conceptos de disyunción y negación; por ejemplo, el axioma $\mathrm{A} 3$ establece que la disyunción es parcialmente conmutativa (v.gr., que si Carlos duerme o Enrique lee, entonces Enrique lee o Carlos duerme). El conjunto (2) formula propiedades fundamentales del concepto de totalidad. El conjunto (3) reduce los indicativos formados con ' $I$ ' a los indicativos descriptivos ordinarios. Son los del conjunto (4) los que más nos interesan, y explico por separado cada uno de estos axiomas:

A14: establece que si la ejecución de un acto es obligatoria, entonces su inejecución no lo es.

A15: se puede leer: "Si es obligatorio hacer juntos dos actos dados, entonces es obligatorio hacer cada uno de ellos por separado, y viceversa."

A16: no tiene una lectura fácil en la lengua ordinaria; es uno de los casos en que la teoría hace más preciso el uso corriente, y quizá hasta lo enriquece; lo que sigue es inexacto, pero da una idea: "Es obligatorio ejecutar un acto $A$ y ciertas circunstancias están presentes únicamente cuando $A$ es obligatorio y esas circunstancias están presentes."

A17: Su traducción tampoco es clara en la lengua corriente: "Es obligatorio: ejecutar un acto que sea obligatorio." A17 no es lo mismo que $K(Z) \rightarrow K(Z)$, que puede leerse: "Es obligatorio ejecutar un acto $A$, si $A$ es obligatorio." Esta última proposición es normativa, pero es un caso particular de una ley general de la lógica ordinaria: "Si $A$ es $B$, entonces $A$ es $B$ ", fórmula que expresa el principio de identidad. ${ }^{17}$

A18: Puede leerse: "Si para todos es obligatorio hacer o recibir un acto $A$, entonces es obligatorio que todos hagan o reciban A." En verdad, el condicional converso también vale, pero es un teorema a demostrar en la sección que sigue: el T.KF.I.

\section{Desarrollo del sistema $N_{1}{ }^{\circ}$.}

En esta sección quiero desarrollar una pequeña parte de $N_{1}{ }^{\circ}$, esto es, quiero demostrar algunas proposiciones o teoremas a efecto de dar una idea más concreta del alcance del sistema. Pero es importante recordar que $N_{1}{ }^{*}$ es una extensión (en el sentido técnico del vocablo), así:

17 Aquí se encuentra una prueba parcial de que la lógica normativa no es paralela a la "lógica del ser" e independiente de ella. Una prueba completa se encuentra en la estructura de $\mathrm{N}_{1}{ }^{*}$, que incluye a la lógica ordinaria como estrato fundamental. 
1. La lógica proposicional se extiende, mediante la regla de formación 2)A)c) y la de universalización y axiomas A5-A7, a una lógica funcional;

2. La logica funcional se extiende, mediante las reglas de formación de imperativos y 2)A)e) y 2)A)g) y los axiomas A8-A13, a una lógica imperativa;

3. La lógica imperativa se extiende, mediante las reglas 2)A)f) y III y los axiomas A14-18, a una lógica normativa. ${ }^{18}$

En consecuencia, $N_{1}{ }^{\circ}$ incluye toda la lógica proposicional y funcional ordinaria, pero no es necesario que desarrollemos todos sus teoremas en este estudio, ya que se encuentran demostrados y ordenados en cualquier libro de lógica simbólica. Pero sí podemos basarnos en cualquier teorema de la lógica proposicional o de la lógica funcional general para nuestras demostraciones.

A. Teoremas de la lógica imperativa. Lo único que hay que demostrar son ciertas propiedades formales de los conectivos imperativos. Para simplificar nuestra exposición utilizaremos las letras ' $X{ }^{\prime},{ }^{\prime} X^{\prime}$, ' $Z$ ' para denotar imperativos, $y$ las letras ' $p$ ', ' $q$ ', ' $r$ ', para denotar indicativos.

T.I.1. Conmutatividad de la disyunción imperativa:

\section{Demostración:}

(1) $I(X \vee Z) \equiv I Y \vee I Z$

(2) $I Y \vee I Z=I Z v I Y$

(3) $I Z v I Y \equiv I(Z v Y)$

(4) $I(Y \vee Z) \equiv I(Z \vee Y)$

$$
I(Y \vee Z) \equiv I(Z \vee Y) \text {. }
$$

por A10

por Conmutatividad de la disyunción indicativa por Al0

por Transitividad del indicativo.

T.I.2. Asociatividad de la disyunción imperativa:

Demostracion:
(1) $I((Z v X) v Y) \equiv I(Z v X) v I Y$
(2) $(I(Z \vee X) v I Y) \equiv(I Z v I X) v I Y$
(3) $(I Z \vee I X) v I Y \equiv I Z v(I X v I Y)$
(4) $I Z v(I X v I Y) \equiv I Z v I(X v Y)$

$$
I((Z v X) v Y) \equiv I(Z v(X v Y)) .
$$

$\begin{aligned} \text { por } & A 10 \\ \text { por } & A 10 \\ \text { por Conmut. } & \text { de } v \text { indicat. } \\ \text { por } & A 10\end{aligned}$

por $\mathrm{A} 10$

mut. de $v$ indicat.

por Al0

18 Esta triple estructura "extensiva" es lo que principalmente distingue a $\mathrm{N}_{1}^{\circ}$ del sistema de von Wright y de la Axiomática de García Máynez. El sistema de von Wright es más comprensivo que la Axiomática de García Máynez, pero comprende menos que $\mathrm{N}_{1}{ }^{\circ}$. El sistema de von Wright es un sistema de normas en el cual sólo hay normas al nivel del cálculo proposicional, es decir, no incluye: (a) imperativos, (b) indicativos descripti. vos u ordinarios, (c) normas mixtas, esto es, que tienen elementos indicativos (v.gr., "Si Juan mata a Pedro, Juan debe ser castigado"), (d) normas con cuantificadores, es decir, en las que entran los términos "todos", "algunos" (v.gr., "Todos deben no matar". "Hay alguien que no tiene prohibido matar", "Todos deben respetar al Alcalde"). Ahora bien, estos cuatro tipos de oraciones aparecen en $\mathrm{N}_{1}{ }^{\circ}$. De otro lado, $\mathrm{N}_{1}{ }^{\circ}$ se distingue del sistema 
(5) $I Z v I(X v Y) \equiv I(Z v(X \vee Y))$

por A10

(6) $I((Z \vee X) v Y) \equiv I(Z \vee(X \vee Y))$

por Transitiv. de $\equiv$.

T.I.3. Conmutatividad de la disyunción mixta: $I(p \circ Z) \equiv I(Z \circ p)$.

Demostración:

A11, A12, y Transitividad de $\equiv$.

T.I.4. Asociatividad de la disyunción mixta: $I((p \vee q) \circ Z) \equiv I(p o(q \circ Z))$. Demostración:

Similar a la de T.I.2 utilizando Al1 en lugar de A10.

T.I.5. Asociatividad de la disyunción mixta:

Demostración:

$$
I((p \circ Y) v Z) \equiv I(p o(Y \vee Z) .
$$

Similar a la anterior utilizando All también.

T.I.6. Descomposición de la conjunción imperativa: $I(Y . Z) \equiv I Y . I Z$. Demostración:

(1) $\quad I(Y . Z) \equiv I-(-Y v-Z)$

(2) $I-(-Y v-Z) \equiv-I(-Y v-Z)$

(3) $-I(-Y v-Z) \equiv-(I-Y v I-Z)$

(4) $-(I-Y v I-Z) \equiv-(-I Y v-I Z)$

(5) $-(-\Gamma \quad v-I Z) \equiv I Y . I Z$

(6) $\quad I(Y, Z) \equiv I Y . I Z$

por Def. 4

por A9

por $\mathrm{A} 10$

por A9

por Def. 4

por Transitividad de $\equiv$.

T.I.7. Conmutatividad de la conjunción imperativa: $I(Y . Z) \equiv I(Z, Y)$. Demostración:

(1) $I(Y . Z) \equiv I Y . I Z$

(2) $\quad Y . I Z \equiv I Z . \Gamma$

(3) $I Z . M \equiv I(Z, Y)$

(4) $I(Y . Z) \equiv I(Z . Y)$

por T.I.6

por Conmutatividad de la conjunción indicativa

por T.I.6

por Transitividad de $\equiv$.

T.I.8. Asociatividad de la conjunción imperativa:

Demostración:

(1) $I((X . Y) \cdot Z) \equiv I(X . Y) \cdot I(Z)$

(2) $I(X . Y) . I Z \equiv(I X . I Y) . I Z$

(3) $(I X . I Y) . I Z \equiv I X .(I Y . I Z)$
$I((X . Y) . Z) \equiv I(X .(Y . Z))$.

por T.I.6

por T.I.6

por Asociatividad de la conjunción indicativa

de von Wright en que en él las normas contienen imperativos. Finalmente, en $\mathrm{N}_{1}{ }^{\circ}$ el término "deber" es el único término normativo indefinible; en el sistema de von Wright lo es el término "ser permitido"; en la Axiomática de García Máynez parece haber cuatro términos normativos básicos: "obligatoriedad", "licitud", "ilicitud" y "optatividad"; cf., por ejemplo, Los principios de la ontología formal del derecho y su expresión simbólica, págs. 33 sigs. 
UN SISTEMA GENERAL DE LÓGICA NORMATIVA
(4) $I X .(I Y . I Z) \equiv I X . I(Y . Z)$
por T.I.6
(5) $I X . I(Y . Z) \equiv I(X .(Y . Z))$
por T.I.6
(6) $I((X, Y) . Z) \equiv I(X .(Y, Z))$ por Transitividad de $\equiv$.

T.I.9 Distributividad de la conjunción a través de la disyunción:

Demostración:

$I(X .(Y \vee Z)) \equiv I(X . Y \vee X Z)$.

(1) $\quad I(X .(Y \vee Z)) \equiv I X . I(Y \vee Z)$

por T.I.6

(2) $I X . I(Y \vee Z) \equiv I X .(I Y \vee I Z)$

por A10

(3) $I X .(I Y v I Z) \equiv I X . I Y v I X . I Z$

(4) $I X . I Y v I X . I Z \equiv I(X, Y) v I(X . Z)$

por Distributividad indic.

(5) $I(X . Y) \vee I(X . Z) \equiv I(X . Y \vee X . Z)$

(6) $I(X .(Y \vee Z)) \equiv I(X . Y \vee X . Z$

por T.I.6

por $\mathrm{A} 10$

por Transitividad de $\equiv$.

T.I.10. Distributividad de la disyunción a través de la conjunción:

Demostración:

$I(X \vee(Y . Z)) \equiv I((X \vee Y) .(X \vee Z))$.

Similar a la anterior, usando primero $\mathrm{Al0}$ y después T.I.6.

T.I.11. Distributividad de la conjunción a través de la disyunción mixta:

Demostración:

$I(X .(p \circ Z)) \equiv I(X \& p \vee X . Z)$.

Similar a las anteriores.

Los siguientes teoremas se dan sin demostración por brevedad:

T.I.12. Conmutatividad de la conjunción mixta: $I(p \& Z) \equiv I(Z \& p)$.

T.I.12a. Descomposición de la conjunción mixta: $I(p \& Z) \equiv p . I Z$.

T.I.13. Distributividad de la disyunción mixta:

$$
I(p \circ(Y . Z)) \equiv I((p \circ Y) \cdot(p \circ Z)) .
$$

T.I.14. Distributividad de la conjunción mixta:

$$
I(p \&(Y v Z)) \equiv I(p \& Y v p \& Z) .
$$

T.I.15. Asociatividad de las conjunciones imperativas:

$$
I(p \&(Y . Z)) \equiv I((p \& Y) . Z) .
$$

T.I.16. Asociatividad de las conjunciones imperativa e indicativa:

$$
I((p \cdot q) \& Z) \equiv I(p \&(q \& Z)) \text {. }
$$

T.I.17. Descomposición del condicional imperativo: $I(Y \rightarrow Z) \equiv I Y \rightarrow I Z$.

T.I.18. Descomposición del condicional mixto: $I(p \rightarrow Z) \equiv p \rightarrow I Z$.

T.I.19. Descomposición del bicondicional imperativo:

$$
I(Y \equiv Z) \equiv(I Y \equiv I Z)
$$


T.I.20. Descomposición del cuantificador existencial imperativo:

$$
I(\exists X)(Z) \equiv(\exists X) I(Z) \text {. }
$$

Nota: En este teorema $X$ es una variable individual. Demostración:
(1) $I(\boldsymbol{B X})(Z) \equiv I-(X)-(Z)$
(2) $I-(X)-(Z) \equiv-(X)-I(Z)$
(3) $-(X)-I(Z) \equiv(B X) I(Z)$
(4) $I(B X)(Z) \equiv(B X) I(Z)$

\author{
por Def. 1 \\ por $A 9, A 13, A 9$ \\ por Def. 1 \\ por Transitividad de $=$.
}

B. Teoremas de la lógica normativa. Aquí podemos distinguir dos estratos:

(1) el de la lógica normativa proposicional, y (2) el de la lógica normativa funcional. En el primero no entran: la regla de formación 2)A)c), los axiomas Al3 y Al8; sin embargo, esta distinción es menos importante que la distinción paralela en que se origina, propia de la lógica indicativa ordinaria, correspondiente al paso del primer conjunto de axiomas al segundo. De todos modos, la señalaremos para facilitar la comparación de $N_{1}^{*}$ con el sistema de von Wright, por ejemplo.

T.KP.1. $K Z \rightarrow K(Z v Y) \quad$ "Si debe hacerse $A$, entonces debe hacerse $A$ o $B . "$

$\begin{array}{ll} & \text { Demostración: } \\ \text { (1) } I Z \rightarrow I Z v I Y & \text { por A2 } \\ \text { (2) } I Z v I Y \equiv I(Z v Y) & \text { por A10 } \\ \text { (3) }(I Z v I Y \rightarrow I(Z v Y)) .(I(Z v Y) \rightarrow I Z v I Y))\end{array}$

(4) $I Z \vee I Y \rightarrow I(Z \vee Y)$

(3), por Simplificación

(2), por Def. 6 (probada en lógica ordinaria)

(5) $I Z \rightarrow I(Z v Y)$

(1), (4); Transitividad

(6) $K Z \rightarrow K(Z v Y)$ del $\rightarrow$ indicativo.

(5); regla III.

T.KP.2. $K Z \rightarrow K(Y \vee Z)$

"Si $A$ debe hacerse, entonces debe hacerse $B$ o $A . " 19$

19 Hace algunos años hubo una disputa famosa sobre si el imperativo "Pon "sict al correo" implicaba el imperativo "Pon esa carta al correo o quémala". Evidentemente, implicación en el sentido de inferencia es en $\mathrm{N}_{1}$ " completamente inaplicable a imperativos; pero T.KP.I puede decirse que de algún modo representa una respuesta afirmativa a la pregunta de la disputa. Pero nótese que no hay ninguna razón para preocuparse. En efecto por T.KP.I tenemos las dos proposiciones: $K Z \rightarrow K\left(\begin{array}{lll}Y & \vee & Z\end{array}\right)$ y $K Z \rightarrow K\left(\begin{array}{lll}-Y & \vee & Z\end{array}\right)$, lo cual nos conduce, según un teorema de lógica general indicativa, a $K Z \rightarrow K(Y \vee Z)$. .$K(-Y \vee Z)$, lo cual nos da por A15 y transitividad de $\rightarrow: K Z \rightarrow K((Y \cup Z) .(-Y \vee Z)), y$ este último consecuente se reduce a $K Z$; de modo que T.KP.1 nos lleva a $K Z \rightarrow K Z$, que es una tautología trivial. Para una solución diferente de esta aparente paradoja. Cf. Hare, "Imperative Sentences", Mind., 1949; págs. 43 sigs. 
Demostración:

Como la anterior utilizando la conmutatividad del $v$ indica tivo después de (1).

T.KP.3. $K Z \rightarrow K(p \rightarrow Z)$

"Si una acción A debe hacerse, entonces debe hacerse $A$, cualquiera que sea el hecho que

Demostración:

(1) $\quad I Z \rightarrow(p \rightarrow I Z)$ ocurra."

(2) $\quad(p \rightarrow I Z) \equiv I(p \rightarrow Z)$

una tautalogía

(3) $\quad$ por T.I.18

(4) $\quad(p \rightarrow I Z) \rightarrow I(p \rightarrow Z)$

por Def. 6

$$
I Z \rightarrow I(p \rightarrow Z)
$$

(6)

$$
K Z \rightarrow K(p \rightarrow Z)
$$

(3), por Simplificación

Nota: Este teorema puede interpretarse como sigue: "Si una acción es obligatoria (a secas, simpliciter), tal acción es condicionalmente obligatoria (además)."

T.KP.4. $K Z \equiv K(p \rightarrow Z) . K(-p \rightarrow Z)$

"Una acción $A$ es obligatoria únicamente si es obligatoria en

Demostración: cualesquiera circunstancias."
(1) $I Z \equiv(p \rightarrow I Z) \cdot(-p \rightarrow I Z)$
una tautología
(2) $(p \rightarrow I Z) \cdot(-p \rightarrow I Z) \equiv I(p \rightarrow Z) \cdot I(-p \rightarrow Z)$
por T.I.18 y A9
(3) $I(p \rightarrow Z) \cdot I(-p \rightarrow Z) \equiv I((p \rightarrow Z) .(-p \rightarrow Z))$
por T.I.6
(4) $I Z \equiv I((p \rightarrow Z) .(-p \rightarrow Z))$
(5) $K Z \equiv K((p \rightarrow Z) \cdot(-p \rightarrow Z))$
(6) $K Z \equiv K(p \rightarrow Z) . K(-p \rightarrow Z)$
Transitividad de $\equiv$.
(4); Regla III
(5); A15.

Esta demostración se acortó un poco gracias a la Regla KII, que demostramos en seguida. De aquí en adelante reuniremos dos o tres pasos en uno (así como hicimos en el paso (2)); por ejemplo: de un bicondicional sacaremos de una vez uno de sus componentes conjuntivos, juntando el paso Def. 6 y la Simplificación.

Regla KII: Si $I Z \equiv I Y$ es demostrable en $N_{1}$ con las mismas restricciones impuestas por Regla III, entonces $K Z \equiv K Y$ es también demostrable en $N_{1}^{*}$.

Demostración:

(1) Si $I Z \equiv I Y$ es demostrable $(I Z \rightarrow I Y) \cdot(I Y \rightarrow I Z)$, también es demostrable (ambos con la mismas restricciones de la Regla III); por Def. 6. 
(2) Si $(I Z \rightarrow I Y)$. $(I Y \rightarrow I Z)$ es demostrable, $I Z \rightarrow I Y$ es también demostrable (idem); por Simplificación.

(3) Si $(I Z \rightarrow I Y) .(I Y \rightarrow I Z)$ es demostrable, entonces $I Y \rightarrow I Z$ es también demostrable (idem); por Simplificación.

(4) Si $I Z \rightarrow I Y$ es demostrable, $K Z \rightarrow K Y$ es demostrable (idem); por Regla III.

(5) Si $I Y \rightarrow I Z$ es demostrable, entonces $K Y \rightarrow K Z$ es también demostrable (idem); por Regla III.

(6) Si $(I Z \rightarrow I Y) .(I Y \rightarrow I Z)$ es demostrable, entonces $K Z \rightarrow K Y$ es también demostrable (idem); de (2) y (4) por Regla III. .

(7) Si $(I Z \rightarrow I Y) .(I Y \rightarrow I Z)$ es demostrable, $K Y \rightarrow K Z$ es también demostrable (idem); de (3) y (5).

(8) Si $(I Z \rightarrow I Y) \cdot(I Y \rightarrow I Z)$ es demostrable $(K Z \rightarrow K Y) \cdot(K Y \rightarrow K Z)$ es demostrable (idem); de (6) y (7).

(9) Si $I Z \rightarrow I Y$ es demostrable, entonces $(K Z \rightarrow K Y) .(K Y \rightarrow K Z)$ es demostrable (idem); de (1) y (9).

(10) Si $I Z \equiv I Y$ es demostrable, entonces $K Z \equiv K Y$ es también demostrable en $N_{1}{ }^{*}$ con las mismas restricciones de la Regla III.

T.KP.5. $K Z \equiv K(Y \rightarrow Z) . K(-Y \rightarrow Z) \quad$ "Una acción $A$ es obligatoria únicamente si es obligatoria a condición de hacer cualquiera acción y dejar de hacer cualquiera acción."

\section{Demostración:}

Como la anterior.

Este teorema es un caso especial de T.KP.4 en el sentido de que este último se refiere a cualquier suceso, el cual puede ser una acción humana.

T.KP.6. $K Z v K Y \rightarrow K(Z v Y)$

"Si es obligatorio hacer $A$ o hacer $B$, entonces es obligatorio ejecutar una de las dos acciones por lo menos."

\section{Demostración:}

(1) $(K Z \rightarrow K(Z \vee Y)) .(K Y \rightarrow K(Z \vee Y)) \rightarrow(K Z \vee K Y)$ $K(Z v Y)$

(2) $K Z \rightarrow K(Z \vee Y)$

(3) $K Z \rightarrow K(Z \vee Y)$

una tautología

(4) $(K Z \rightarrow K(Z \vee Y)) .(K Y \rightarrow K(Z \vee Y))$

por T.KP.1

(5) $K Z v K Y \rightarrow K(Z v Y)$

por T.KP.2

(2), (3); Conjunción

(1), (4); Regla I.

T.KP.7 $K(Z v Y) \rightarrow-K-Z v K Y$

"Si es obligatorio escoger entre dos acciones $A$ y. $B$, entonces 
una es obligatoria o la no ejecución de la otra es obliga. Demostración:

(1) $(I Z \vee I Y),-I Z \rightarrow I Y$

(2) $(I Z v X) \cdot-I Z \equiv I(Z v Y),-I Z$

(3) $\quad I(Z \vee Y) .-I Z \equiv I((Z \vee Y) .-Z)$

(4) $I((Z \vee Y) .-Z) \rightarrow I Y$ toria."

(5) $\quad K((Z \vee Y) .-Z) \rightarrow K Y$

(6) $K(Z \vee Y) \cdot K(-Z) \rightarrow K Y$

(7) $K(Z \vee Y) \rightarrow(K-Z \rightarrow K Y)$

(8) $K(Z \vee Y) \rightarrow-K-Z v K Y$

una tautología por Al0 por A9, T.I.6

sustituyendo en (1) conforme a (3)

(4); Regla III

(5); por A15

(6); por Exportación

(7); por Def. 2.

Ahora vamos a introducir algunas definiciones normativas muy valiosas:

Def. 7.

$P(Z)=$ Def. $-K(-Z)$.

Def. 8. $\quad F(Z)=$ Def. $K(-Z)$.

Def. $9 . \quad L(Z)=$ Def. $-K(Z) .-K(-Z)$.

Como $K$ expresa obligatoriedad o deber, el sentido de estas definiciones resulta bastante claro:

7. Un acto está permitído si y sólo si su no ejecución no es obligatoria.

8. Un acto está prohibido únicamente si su no ejecución es obligatoria.

9. Un acto es libre (completamente) únicamente si no es obligatoria su ejecución ni su no ejecución.

T.KP.8. $K(Z \vee Y) \rightarrow(F Z \rightarrow K Y)$

"Si es obligatorio escoger entre dos actos, entonces: si uno está pro-

Demostración: hibido el otro es obligatorio."

(1)-(7) como en la demostración anterior.

(8) aplíquese la Def. 8 a (7).

T.KP.9. $K(Z \rightarrow Y) \rightarrow(K Z \rightarrow K Y)$

"Si es obligatorio: hacer un acto $A$ con la condición de otro acto $B$, entonces si $B$ es obligatorio, $A$ también lo es."

\section{Demostración:}

(1)-(7) como en la demostración de T.KP.7.

(8) Substitúyase $-Z$ por $Z$ en (7): $K(-Z \vee Y) \rightarrow(K Z \rightarrow K Y)$

$K(Z \rightarrow Y) \rightarrow(K Z \rightarrow K Y)$

(8); por Def. 2. 
T.KP.10. $K(Z \rightarrow Y) \rightarrow(-K Y \rightarrow-K Z)$

Demostración:
(1) $K(Z \rightarrow Y) \rightarrow(K Z \rightarrow K Y)$
(2) $K(Z \rightarrow Y) \rightarrow(-K Y \rightarrow-K Z)$

T.KP.II. $K(Z \rightarrow Y) \rightarrow(F(Y) \rightarrow F(Z))$

Demostración:

(1) $I(Z \rightarrow Y) \rightarrow(I Z \rightarrow I Y)$

(2) $(I Z \rightarrow I Y) \rightarrow(-I Y \rightarrow-I Z)$

(3) $(-I Y \rightarrow-I Z) \rightarrow I(-Y \rightarrow-Z)$

(4) $I(Z \rightarrow Y) \rightarrow I(-Y \rightarrow-Z)$

(5) $K(Z \rightarrow Y) \rightarrow K(-Y \rightarrow-Z)$

(6) $K(-Y \rightarrow-Z) \rightarrow(K-Y \rightarrow K-Z)$

(7) $K(-Y \rightarrow-Z) \rightarrow(F Y \rightarrow F Z)$

(8) $K(Z \rightarrow Y) \rightarrow(F Y \rightarrow F Z)$

T.KP.12 $K(Z \rightarrow Y) \rightarrow(P Z \rightarrow P Y)$
"Si un acto $A$ es obligatorio bajo condición de otro acto $B$, entonces si $A$ no es obligatorio, $B$ tampoco lo es."

\section{por T.KP.9}

(1); por contraposición

"Si un acto es obligatorio bajo condición de que se haga otro acto, entonces si éste está prohibido, aquél también lo está."

por T.1.17 una tautología por T.I.17, A9.

(1), (3); Transitividad de $\rightarrow$. (4); Regla III.

T.KP.9.

(6); por Def. 8.

(5), (7); Transit. de $\rightarrow$.

"Si un acto es obligatorio bajo condición de que otro acto sea ejecutado, entonces si éste está permitido, aquél también está permitido."

Demostración:

(1)-(6) como en la anterior.

(7) $K(Z \rightarrow Y) \rightarrow(K-Y \rightarrow K-Z)$

(8) $(K-Y \rightarrow K-Z) \rightarrow(-K-Z \rightarrow-K-Y)$

(5), (6); Transitiv. de $\rightarrow$.

(9) $K(Z \rightarrow Y) \rightarrow(-K-Z \rightarrow-K-Y)$ una tautología.

(10) $K(Z \rightarrow Y) \rightarrow(P Z \rightarrow P Y)$

(7), (8); Transitiv. de $\rightarrow$. (9); Def. 7.

T.KP.13. $-K(Z .-Z)$

"La ejecución y la inejecución de un mismo acto no son juntamente obligatorias."

Demostración:

(1) $K Z \rightarrow-K-Z$

(2) $-(K Z \cdot K-Z)$

(3) $-K(Z,-Z)$

T.KP.14. $P(Z . Y) \rightarrow P Z$
A14.

(1); paso de condicional a conjunción.

(2); por A15.

"Si un acto doble está permitido, cada parte de él está permitida." 
Demostración:
(1) $-I Z \rightarrow(-I Z v-I Y)$
una tautología
(2) $I-Z \rightarrow I(-Z v-Y)$
(1); A9, A10.
(3) $K-Z \rightarrow K(-Z v-Y)$
(2); Regla III.
(4) $K-Z \rightarrow K(-(Z . Y))$
(3); paso de la disyunción a con- junción; doble negación
(5) $-K(-(Z . Y)) \rightarrow-K-Z$
(6) $P(Z, Y) \rightarrow P Z$
(4); contraposición
(5); def. 7 .

T.KP.15. $K(p \rightarrow Z) \rightarrow(p \rightarrow K Z)$

"Si un acto es obligatorio bajo condición de que un hecho suceda, entonces si éste sucede el acto es obligatorio simpliciter."

Demostracion:

(1) $(p \rightarrow I Z) \cdot p \rightarrow I Z$

(2) $I(p \rightarrow Z) \cdot p \rightarrow I Z$

(3) $I((p \rightarrow Z) \& p)) \rightarrow I Z$

(4) $K((p \rightarrow Z) \& p)) \rightarrow K Z$

(5) $K(p \rightarrow Z) \cdot p \rightarrow K Z$

(6) $K(p \rightarrow Z) \rightarrow(p \rightarrow K Z)$

Una tautología.

(1); T.I.18.

(2);T.12.a.

(3); Regla III.

(4); A15, conmutación.

(5); Exportación.

T.KP.16. $K(p \circ Z) \rightarrow p v K Z$

Demostración:

(1) $K(-p \rightarrow Z) \rightarrow(-p \rightarrow K Z)$

(2) $K(p \circ Z) \rightarrow(p \vee K Z)$

T.KP.15.

(1); Def. 2; doble negación.

T.KP.17. $K(K Z \circ Z) \equiv K Z$

"Un acto es obligatorio únicamente si es obligatorio que se haga o el acto es obligatorio."

Demostración:

(1) $K(K Z \circ Z) \rightarrow K Z v K Z$

(2) $K Z \cup K Z \rightarrow K Z$

(3) $K(K Z \circ Z) \rightarrow K Z$

(4) $K Z \rightarrow K(-K Z \rightarrow Z)$

(5) $K Z \rightarrow K(K Z \circ Z)$

T.KP.16.

tautología

(1), (2); Transitividad de $\rightarrow$. T.KP.3.

(4); Def. 2; doble negación

(6) $(K(K Z \circ Z) \rightarrow K Z) .(K Z \rightarrow K(K Z \circ Z)) \quad$ (3), (5); Conjunción

(7) $K(K Z \circ Z) \equiv K Z$

(6); Def. 6.

T.KP.18. $K Z \rightarrow P Z$

"Si un acto es obligatorio, está permitido."

Deniostración:

Substitución de $P$ por $-K-$ en A14; Def. 7.

T.KP.19. $P Z v P-Z$

"O la ejecución de un acto está permitida o está permitida su no ejecución." 
Demostración:
(1) $-K(Z) v-K(-Z)$
(2) $-K(-Z) v-K(-Z)$
(3) $\quad P-Z v P Z$
(4) $\quad P Z \cup P-Z$

T.KP.20. $L Z \equiv-K Z .-F Z$

Demostración:
(1) $L Z \equiv-K Z \cdot-K-Z$
(2) $L Z=-K Z .-F Z$

T.KP.21. $L Z \equiv P Z, P-Z$
A14; Def. 2.

(1); doble negación.

(2); Def. 7 .

(3); conmutatividad de $v$.

"Un acto es (completamente) libre si y sólo si no está prohibido ni es obligatorio."

Demostración:

(1) $L Z \equiv-F Z$. $-K--Z$

(2) $L Z \equiv-K-Z,-K--Z$

(3) $L Z \equiv P Z . P-Z$

Def. 9.

(1); Def. 8.

"Un acto es (completamente) libre únicamente si tanto su ejecución como su no ejecución están permitidas."
T.KP.20; doble negación y con- mutatividad de $v$.
(1); Def. 8.
(1); Def. 7 .

T.KP.22. $K Z \equiv P Z . F-Z$

"Un acto es obligatorio únicamente si su ejecución está permitida y su no ejecución está prohibida."

Demostración:
(1) $K(--Z) \equiv F-Z$
(2) $K(Z) \rightarrow F-Z$
(3) $K Z \rightarrow P Z$
(4) $K Z \rightarrow P Z, F-Z$
(5) $P Z . F-Z \rightarrow F-Z$
(6) $P Z$. F $-Z \rightarrow K Z$
(7) $K Z \equiv P Z \cdot P-Z$

T.KP.23. $F Z \equiv-P Z$

Demostración:

(1) $K-Z \equiv-(-K-Z)$

(2) $\mathrm{FZ} \equiv-\mathrm{PZ}$

Def. 8; doble negación

(1); simplificación

T.KP.18.

(2), (3); combinación

una tautología

(5); substitución conforme a

(1); doble negación.

(4), (6); conjunción y Def. 6 .

"Un acto está prohibido únicamente si no está permitido."

Principio de doble negación. (1); Def. 8 y Def. 7.

T.KP.24. Corolario de T.KP.23. $-(P Z . F Z)$

T.KP.25. $F Z \rightarrow F(Z . Y)$

"Si un acto $A$ está prohibido, también 
Demostración:

está prohibido cualquier acto complejo que tenga a $A$ como parte."

(1) -(4) de la demostración de T.KP.14: $K-Z \rightarrow(-(Z . Y))$

(5)

$F Z \rightarrow F(Z . Y)$

(4); Def. 8.

T.KP.26. $K(Y \vee Z) \equiv K(Z \vee Y) \quad$ Conmutatividad interna de $v$ imperativo.

Demostración:

(1) $I(Y \vee Z) \equiv I(Z v Y)$

"Debe escogerse entre dos actos $A$ y $B$ únicamente si debe escogerse entre B y A."

(2) $K(Y \vee Z) \equiv K(Z \vee Y)$

T.I.1.

(1); Regla K II.

Los teoremas que siguen tienen una demostración semejante a la última: T.KP.27. $K(Z . Y) \equiv K(Y . Z)$ "Debe hacerse $A$ y $B$ cuando y sólo cuando debe hacerse $B$ y $A . "$

T.KP.28. $K((Z \vee Y) v X) \equiv K(Z v(Y \vee X))$

T.KP.29. $K(p \circ Z) \equiv K(Z \circ p)$

T.KP.30. $K(Z .(Y v X)) \equiv K(Z . Y v Z . X)$

T.KP.31. $K(p \&(Y \vee X)) \equiv K(p \& Y v p \& X)$

T.KP.32. $K(Z v Y . X) \equiv K((Z v Y) \cdot(Z v X))$

T.KP.33, $K(p \circ X, Y) \equiv K((p \circ X) \cdot(p \circ Y))$

T.KP.34. $K(p \circ q \& Z) \equiv K((p \vee q) \&(p \circ Z))$

T.KP.35. $K(p \& Z) \equiv K(Z \& p)$

Etcétera.

T.KP.36. $P(Z . Y)=P(Y . Z)$

"Si está permitido escoger entre dos actos $A$ y $B$, entonces está permitido escoger entre $B$ y $A$, y viceversa."

Demostracion:

(1) $K(-Z v Y) \equiv K(-Y v-Z)$

(2) $-K(-Z v-Y) \equiv-K(-Y v-Z)$

(3) $\quad P-(-Z v-Y) \equiv P-(-Y v-Z)$

(4) $\quad P(Z . Y) \equiv P(Y . Z)$

T.KP.26.

(1); propiedad de $\equiv$.

(2); Def. 7.

(3); Def. 4.

T.KP.37. $P(Z \vee Y) \equiv P(Y \vee Z)$

T.KP.38. $P((Z \vee Y) \vee X) \equiv P(Z \vee(Y \vee X))$ 
T.KP.39. $P(Z .(Y \vee X)) \equiv P(Z . Y . v Z . X)$

T.KP.40. $P(p \&(Y \vee X)) \equiv P(p \& Y v p \& X)$

T.KP.41. $P(Z \vee(Y . X)) \equiv P(\langle Z \vee Y\rangle) .(Z \vee Y)$.

Etcétera.

T.KP.42. $F(Z . Y) \equiv F(Y . Z)$

"Está prohibido hacer $A$ y $B$ juntos únicamente si está prohibido ha-

Demostración:

(1) $K(-Z v-Y) \equiv K(-Y v-Z) \quad$ T.KP.26.

(2) $K \rightarrow(Z . Y) \equiv K(\rightarrow Z v \rightarrow Y) \quad$ Def. 4; doble negación

(3) $K(\rightarrow Y v \rightarrow Z) \equiv K \rightarrow(Y . Z) \quad$ Def.4; doble negación

(4) $K \rightarrow(Z . Y) \equiv K \rightarrow(Y . Z) \quad(2),(1),(3)$; Transitividad de $\equiv$.

(5) $F(Z . Y) \equiv F(Y . Z) \quad$ (4); Def. 8.

T.KP.43. $F(Z \vee Y) \equiv F(Y \vee Z)$

T.KP.44. $F((Z \vee Y) \cup X) \equiv F(Z \vee(Y \vee X))$

T.KP.45. $F(Z .(Y \vee X)) \equiv F(Z . Y \vee Z . X)$

T.KP.46. $F(Z v Y . X) \equiv F((Z v Y) .(Z v))$

T.KP.47. $F(p o(q \& Z)) \equiv F((p \circ q) \&(p \circ Z))$

T.KP.48. $F(p \&(Z v Y)) \equiv F(p \& Z v p \& Y)$

Etcétera.

T.KP.49. $L(Z, Y) \equiv L(Y . Z) \quad$ "Hay libertad completa de hacer $A$ y $B$ si y sólo si hay libertad completa de hacer $B$ y $A . "$

Demostración:

(1) $L(Z, Y) \equiv P(Z, Y) . P \rightarrow(Z, Y) \quad$ T.KP.21.

(2) $P(Z, Y) . P-(Z . Y) \equiv P(Z, Y) . P(-Z v-Y) \quad$ Def. 4; doble negación

(3) $P(Z . Y) \cdot P(-Z v-Y) \equiv P(Y . Z) \cdot P(-Y v-Z) \quad$ T.KP.36 y T.KP.37.

(4) $P(Y . Z) \cdot P(-Y v-Z) \equiv P(Y . Z) \cdot P-(Y . Z)$ (3); Def. 4, do-

(5) $P(Y . Z) \cdot P-(Y . Z) \equiv L(Y . Z)$ T.KP.21.

$$
L(Z . Y) \equiv L(Y . Z)
$$

(1)-(5); Transitividad de $\equiv$

T.KP.50. $L(Z \vee Y) \equiv L(Y \vee Z)$

T.KP.51. $L(p \& Z) \equiv L(Z \& Z)$

T.KP.52. $L(Z .(Y \vee X)) \equiv L(Z . Y \vee Z . X)$

T.KP.53. $L((Z \vee Y) \vee X) \equiv L(Z v(Y \vee X))$ 
T.KP.54. $L((Z . Y) . X) \equiv L(Z .(Y . X))$

T.KP.55. $L(p \&(Y \vee X)) \equiv L(p \& Y \vee p \& X)$

T.KP.56. $L(p \circ Y . Z) \equiv L((p \circ Y) \cdot((p \circ Z)) \quad$ Etcétera.

T.KP.57. $P(Z, Y) \rightarrow P Z$.PY "Si está permitido hacer un acto compuesto de dos actos simples, cada uno de éstos está separadamente permitido."

Demostración:

(1) $P(Z . Y) \rightarrow P Z$

(2) $P(Z . Y) \rightarrow P(Y . Z)$

(3) $P(Y . Z) \rightarrow P Y$

(4) $P(Z, Y) \rightarrow P Y$

(5) $P(Z, Y) \rightarrow P Z . P Y$

T.KP.58. $P Y \vee P Z \equiv P(Y \vee Z)$
T.KP.14.

T.KP.36; simplificación.

T.KP.I4.

(2), (3); Transitividad de $\rightarrow$.

(1), (4); reunión tautolbgica.

"Está permitido hacer $A$ o está permitido hacer $B$ únicamente si está permitido escoger entre $A$ y $B$."

\section{Demostración:}

(1) $K(-Y \cdot-Z) \equiv K-Y \cdot K-Z$

A15

(2) $-(K-Y \cdot K-Z) \equiv-K(-Y \cdot-Z)$

(3) $-K-Y v-K-Z \equiv-K-(Y \vee Z)$

(1); propiedad de $\equiv$.

(4) $\quad P Y \vee P Z \equiv P(Y \vee Z)$

(2); Def. 4 y doble negación.

(3); Def. 7 .

T.KP.59. $F(Y \cup Z) \equiv F Y . F Z$ "Está prohibido escoger entre dos acciones Demostración: únicamente si cada una de ellas está (separadamente) prohibida."

(1) $K(-Y .-Z) \equiv K-Y . K-Z$

por A15.

(2) $K-(Y \vee Z) \equiv K-Y . K-Z$

(3) $F(Y \vee Z) \equiv F Y \cdot F Z$

(1); Def. 4, doble negación.

(2); Def. 8 .

T.KP.60. FY $v F Z \quad F(Y . Z)$

"Si está prohibido hacer $A$ o está prohibido hacer $B$, entonces está prohibido hacer $A$ y $B$ juntos."

\section{Demostración:}

(1) $F Y \quad F(Y . Z)$

(2) $F Z \quad F(Y . Z)$

T.KP.25.

T.KP.25, T.KP.42.

(3) FY $\cup F Z \quad F(Y . Z)$

(1), (2); combinación tautológica.

T.KP.61. $L Y \equiv L-Y$

"Un acto es (completamente) libre si y sólo si su no ejecución es (completamente) libre." 


\section{Demostracion:}

(1) $L Y \equiv-K Y \cdot-K-Y$

(2) $L-Y \equiv-K-Y .-K-(-Y)$

(3) $L-Y \equiv-K-Y .-K Y$

(4) $L Y \equiv L-Y$

Def. 9.

Def. 9 .

(3); doble negación

(1), (3); Conmutatividad de la conjunción y Transitividad de $\equiv$.

T.KP.62. $L(Y . Z) \rightarrow L Y . P Z v L Z . P Y$

"Si un acto complejo es (completamente) libre, uno de sus dos componentes es (completamente) libre y el otro está

Demostración: permitido, o al revés."

(1) $L(Y . Z) \rightarrow P(Y . Z) . P-(Y . Z) \quad$ T.KP.21.

(2) $P(Y . Z) \rightarrow P Y . P Z$ T.KP.57.

(3) $P(Y . Z) . P-(Y . Z) \rightarrow P Y . P Z . P-(Y . Z)$

(2); por lógica general.

(4) PY.PZ.P-(Y.Z) $\rightarrow P Y . P Z . P(-Y v-Z)$

(3); Def. 4, doble negación.

(5) PY.PZ.P(-Yv-Z) $\rightarrow P Y . P Z .(P-Y v P-Z)$ (4) T.KP. 58

(6) $P Y . P Z .(P-Y \vee P-Z) \rightarrow P Y . P Z . P-Y \vee P Y . P Z . P-Z$

(5); distribución de la conjunción.

(7) $P Y . P Z . P-Y \vee P Y . P Z . P-Z \rightarrow(P Y . P-Y) \cdot P Z v(P Z . P-$ $-Z) \cdot P Y$ conmutatividad de $v$.

(8) (PY.P-Y).PZv(PZ.P-Z).PY $\rightarrow L Y . P Z v L Z . P Y \quad$ Def.9.

(9) $L(Y . Z) \rightarrow L Y . P Z v L Z . P Y \quad$ (1), (3)-(8); Transitividad de $\rightarrow$.

T.KP.63. $K(Z \rightarrow Y) . K(Y \rightarrow X) \rightarrow K(Z \rightarrow X)$

"Si un acto es obligatorio bajo condición de que se haga otro, y es a su vez el primero condición de la obligatoriedad de un tercero, entonces el segundo es condición de la obligatoriedad del tercero."

Demostración:
(1) $(I Z \rightarrow I Y) \cdot(I Y \rightarrow I X) \rightarrow(I Z \rightarrow I X)$
una tautología
(2) $I(Z \rightarrow Y) . I(Y \rightarrow X) . \rightarrow I(Z \rightarrow X)$
(1); T.I.17.
(3) $I((Z \rightarrow Y) \cdot(Y \rightarrow X)) \rightarrow I(Z \rightarrow X)$
(2); T.I.6. 
(4) $K((Z \rightarrow Y) \cdot(Y \rightarrow X)) \rightarrow K(Z \rightarrow X)$

(5) $K(Z \rightarrow Y) \cdot K(Y \rightarrow X) \rightarrow K(Z \rightarrow X)$

(3); Regla III.

(4); A15.

T.KP.64. $(p \rightarrow q) . K(q \rightarrow Y) \rightarrow(p \rightarrow Y)$

Demostración:

"Si un acto $A$ es obligatorio con relación a un hecho y cuando otro hecho ocurre ese hecho también ocurre, entonces el acto $A$ es también obligatorio con relación a ese otro hecho."

Como la anterior utilizando T.I.18 en lugar de T.I.17, T.I.12a en lugar de T.I.6, y A16 en lugar de A15.

T.KP.65. $K(p \rightarrow Z) . K(Z \rightarrow Y) \rightarrow K(p \rightarrow Y)$

"Si un acto es obligatorio en relación con un hecho y con relación a ese acto otro acto es obligatorio, entonces este último acto es obligatorio con relación a tal hecho."

Demostración:

Parecida a las anteriores.

Los 65 teoremas anteriores constituyen una ilustración adecuada de la naturaleza del sistema $N_{1}{ }^{*}$ en cuanto cálculo proposicional normativo. Estos teoremas son apenas unos de los más simples. Pasamos, pues, a demostrar algunos de los teoremas más sencillos del cálculo funcional contenido en $N_{1}{ }^{\circ}$. Usaremos las letras ' $x$ ', ' $y$ ' (minúsculas) autónomamente, es decir, para representar las variables individuales de $N_{1}{ }^{*}$ (que son esas mismas letras).

T.KF.l. $K(x)(Z) \equiv(x) K(Z) \quad$ "Es obligatorio que todos hagan o a todos se haga una acción $A$ únicamente si todos tienen la obligación o frente a todos se tiene la obligación de hacer $A . "$

Demostración:

(1) $(x) K(Z) \rightarrow K(x)(Z)$

A18.

(2) $(x) I(Z) \rightarrow I(Z)$

(3) $I(x)(Z) \rightarrow I(Z)$

A7, substituyendo $x$ por $x$.

(4) $K(x)(Z) \rightarrow K(Z)$

(2); Al3.

(5) $\quad(x)(K(x)(Z) \rightarrow K(Z))$

(3); Regla III.

(6) $K(x)(Z) \rightarrow(x) K(Z)$

(4); Regla II.

(5); distribución de $(x)$ cuando en el antecedente no ocurre $x$ libre.

(7) $((x) K(Z) \rightarrow K(x)(Z)) .(K(x)(Z) \rightarrow(x) K(Z)) \quad$ (1), (6); con-

(8) $K(x)(Z) \equiv(x) K(Z) \quad$ (7); Def. 6 . junción. 
T.KF.2. $P(\exists x)(Z) \equiv(\exists x) P(Z)$ "Está permitido que alguien haga o se le haga una acción $A$ si y sólo si hay alguien que tiene permitido o a quien Demostración: está permitido hacer $A . "$

(1) $K(x)(-Z) \equiv(x) K(-Z)$

T.KF.1.

(2) $-K(x)(-Z) \equiv-(x) K(-Z) \quad$ (1); propiedad de $\equiv$.

(3) $P-(x)-(Z) \equiv-(x)-P-(-Z)(2)$; Def. 7 , doble negación.

(4) $P(\underline{B} x)(Z) \equiv(-x) P-C(Z)$

(3); Def. 1.

(5) $P(\exists x)(Z) \equiv(\exists x) P(Z)$

(4); doble negación.

Regla de inferencia KIII: Si $I Y \rightarrow I Z$ es demostrable conforme a las restricciones impuestas por la Regla III, entonces $P Y \rightarrow P Z$ es también demostrable en $N_{1}{ }^{\circ}$.

Demostración:

(1) Si $I Y \rightarrow I Z$ es demostrable en $N_{1}$ * con las restricciones impuestas por la Regla III, entonces - $I Z \rightarrow-I Y$ es también demostrable en $N_{1}{ }^{*}$ con las mismas restricciones; contraposición.

(2) $\mathrm{Si}-I Z \rightarrow-\Pi Y$ es demostrable con tales restricciones en $N_{1}{ }^{*}$, también $I-Z \rightarrow I-Y$ es demostrable con las mismas restricciones en $N_{1}^{*}$; por axioma A9.

(3) Si $I-Z \rightarrow I-Y$ es demostrable con esas restricciones en $N_{1}{ }^{\circ}$, entonces $K-Z \rightarrow K-Y$ es demostrable en $N_{1}{ }^{*}$; Regla III.

(4) Si $K-Z \rightarrow K-Y$ es demostrable en $N_{1}^{*}$, también lo es su contrapositiva $-K-Y \rightarrow-K-Z$.

(5) $\mathrm{Si}-K-Y \rightarrow-K-Z$ es demostrable en $N_{1}{ }^{*}$, entonces es demostrable en $N_{1}^{*} P Y \rightarrow P Z$; por Definición 7 .

(6) Luego, si $I Y \rightarrow I Z$ es demostrable en $N_{1}^{*}$ con las restricciones impuestas por la Regla III, $P Y \rightarrow P Z$ también es demostrable en $N_{1}^{*}$; de (1) a (5).

T.KF.3. $P(x)(Z) \rightarrow(x) P(Z) \quad$ "Si está permitido que todos hagan o a todos se haga una acción $A$, entonces todos tienen permitido hacer o que les hagan A."

Demostración:

(1) $(x) I(Z) \rightarrow I(Z)$

(2) $I(x)(Z) \rightarrow I(Z)$

A7.

(3) $P(x)(Z) \rightarrow P(Z)$

(1); A13.

(4) $(x)(P(x)(Z) \rightarrow P(Z))$

(2); Regla KIII.

(5) $P(x)(Z) \rightarrow(x) P(Z)$

(3); Regla II.

(4); distribución de $(x)$ cuando $x$ no ocurre libre en antecedente. 
T.KF.4 $(x) K(Z) \rightarrow K($ H $B x)(Z)$ "Si hay alguien obligado a hacer o a que le hagan $A$, entonces es obligatorio que alguien haga o que hagan a alguien $A . "$

Demostración:

(1) $P(x)-Z \rightarrow(x) P-Z$

(2) $-K-(x)-Z \rightarrow(x)-K--Z$

\section{T.KF.3.}

(3) $-(x)-K Z \rightarrow K-(x)-Z$

(1); Def. 7.

(2); contraposición y doble

(4) $(\exists x) K(Z) \rightarrow K(\exists x)(Z)$ negación.

(3); Def. 1.

T.KF.5. $(x) F(Z) \equiv F(\exists x)(Z)$ "Todos tienen prohibido hacer o recibir la acción $A$ si y sólo si está prohibido que Demostración:
(1) $(x) K(-Z) \equiv K(x)(-Z)$
(2) $(x) K-(Z) \equiv K-(\exists x)(Z)$
(3) $(x) F(Z) \equiv F(\mathcal{A} x)(Z)$
T.KF.I.
(1); Def. 1, y doble negación. (2); Def. 8 .

T.KF.6. $(\exists x) F(Z) \rightarrow F(x)(Z)$

"Si hay alguien que tenga prohibido hacer o recibir la acción $A$, entonces está prohibido que todos hagan o reciban $A . "$

\section{Demostración:}

(1) $-(x) P(Z) \rightarrow-P(x)(Z)$

(2) $(I x)-P(Z) \rightarrow-P(x)(Z)$

(3) $($ G $x) F(Z) \rightarrow F(x)(Z)$

T.KF.3; contraposición.

(1); Def. 1 doble negación.

(2); T.KP.23.

T.KF.7. $F(Z x)(Z) \rightarrow F(x)(Z)$ "Si está prohibido que alguien haga o reciba la acción $A$, entonces está proDemostración:

(1) $\mathrm{F}(\exists x)(Z) \rightarrow(x) F(Z)$ hibido que todos hagan o reciban $A . "$

(2) $(x) F(Z) \rightarrow(\exists x) F(Z)$

(3) $(\exists x) F(Z) \rightarrow F(x)(Z)$

(4) $F(\exists x)(Z) \rightarrow F(x)(Z)$

T.KF.5; simplificación lógica funcional general. T.KF.6.

T.KF.8. $L(x)(Z) \rightarrow(x) P(Z)$. ( $(x) P(-Z)$ "Si es (completamente) libre para todos el hacer o recibir $A$, entonces todos tienen permitido hacer o recibir $A$ $y$ alguien tiene permitido no ejecutar o no recibir $A . "$ 


\section{Demostración:}

(1) $L(x)(Z) \rightarrow P(x)(Z) \cdot P-(x)(Z)$

(2) $P(x)(Z) \rightarrow(x) P(Z)$

Def. 9; simplificación.

(3) $\quad P(x)(Z) \cdot P-(x)(Z) \rightarrow(x) P(Z) . P-(x)(Z)$ T.KF.3.

(4) $P(x)(Z) \cdot P-(x)(Z) \rightarrow(x) P(Z) \cdot P(\exists x)(-Z)$

(2); lógica general.

(3); Def. 1; doble negación.

(5) $P(x)(Z) . P-(x)(Z) \rightarrow(x) P(Z) .(B x) P(-Z)$ (4) T.KF. 2

(6) $L(x)(Z) \rightarrow(x) P(Z) .(\exists x) P(-Z)$

(1), (5); Transitividad de $\rightarrow$.

T.KF.9. $K Y \rightarrow K(\exists x) Z$, siendo $Y$ igual que $Z$ excepto en que $Y$ contiene una variable individual diferente de $x$ o un nombre propio en los lugares en que $Z$ contiene ocurrencias libres de $X$.

Ejemplo: Si Carlos debe hacer o recibir $A$, entonces es obligatorio que alguien haga o reciba $A$.

Demostración:

(1) (x)I-Z $\rightarrow I-Y$, siendo $Y$ igual a $Z$.

(2) $I(x)-Z \rightarrow I-Y$ excepto en que...

A7; $A 9$.

(3) $-I-Y \rightarrow-I(x)-Z$

(1); A13.

(4) $I-Z \rightarrow I-(x)-Z$

(2); contraposición.

(5) $I Y \rightarrow I(\pi x) Z$

(6) $K Y \rightarrow K(\exists x) Z$

(3); A9.

(4); Def. 1, doble negación.

(5); Regla III.

T.KF.10. $K(x)(f \rightarrow Z) \rightarrow(x)(f \rightarrow K Z)$ "Si es obligatorio que todos hagan o reciban $A$ en aconteciendo un hecho dado, entonces para todos vale que si este hecho acontece $A$ es obligatoria."

\section{Demostración:}

(1) $K(x)(f \rightarrow Z) \rightarrow(x) K(f \rightarrow Z) \quad$ T.KF.1.

(2) $K(f \rightarrow Z) \rightarrow(f \rightarrow K Z) \quad$ T.KP.15.

(3) $(x)(K(f \rightarrow Z) \rightarrow(f \rightarrow K Z)) \quad$ (2); Regla II.

(4) $\quad(x)(K(f \rightarrow Z) \rightarrow(f \rightarrow K Z)) \rightarrow((x) K(f \rightarrow Z) \rightarrow(x)(f \rightarrow K Z))$

(5) $(x) \mathrm{K}(f \rightarrow Z) \rightarrow(x)(f \rightarrow K Z)$ lógica funcional general.

(6) $K(x)(f \rightarrow Z) \rightarrow(x)(f \rightarrow K Z)$

(3), (4); Regla I o modus ponens.

(1), (5); Transitividad de $\rightarrow$.

T.KF.11. $K(x)(Y \rightarrow Z) \rightarrow(K(x) Y \rightarrow K(x) Z) \quad$ "Si es obligatorio que todos hagan o reciban $B$ en haciéndose $A$, entonces si 
es obligatorio que todos hagan o reciban $A$, es también obligatorio que Demostración:

(1) $(x)(I Y \rightarrow I Z) \rightarrow((x) I Y \rightarrow(x) I Z)$

(2) $(x)(I Y \rightarrow I Z) \rightarrow(I(x) Y \rightarrow I(x) Z)$ todos hagan o reciban $B$."

(3) $I(x)(Y \rightarrow Z) \rightarrow I((x) Y \rightarrow(x) Z)$

lógica funcional general.

(4) $K(x)(Y \rightarrow Z) \rightarrow K((x) Y \rightarrow(x) Z)$

(1); Al3.

(2); T.I.17, Al3.

(3); Regla III.

(5) $K((x) Y \rightarrow(x) Z) \rightarrow(K(x) Y \rightarrow K(x) Z)$

(6) $K(x)(Y \rightarrow Z) \rightarrow(K(x) Y \rightarrow K(x) Z)$

T.KP.9.

(4), (5); Transitividad de $\rightarrow$.

T.KF.12. $K(x)(Y \rightarrow Z) \rightarrow(F(x) Z \rightarrow F(x) Y)$

Demostración:

Como la anterior, pero con una transposición en el consecuente como segundo paso; así tenemos:

(7) $K(x)(Y \rightarrow Z) \rightarrow(K-(x) Z \rightarrow K-(x) Y)$

(8) $K(x)(Y \rightarrow Z) \rightarrow(F(x) Z \rightarrow F(x) Y)$

(7); Def. 8.

T.KF.13. $K(x)(Y \rightarrow Z) \rightarrow(P(x) Y \rightarrow P(x) Z)$ "Si es obligatorio que todos hagan o reciban $B$ en haciéndose $A$, entonces si está permitido que todos hagan o reciban $A$, está permitido que todos hagan o reciban B."

\section{Demostración:}

Como las anteriores.

(1)-(7) como en la demostración de T.KF.12.

(8) $K(x)(Y \rightarrow Z) \rightarrow(-K-(x) Y \rightarrow-K-(x) Z) \quad$ (7); Transposición en el consecuente.

(9) $K(x)(Y \rightarrow Z) \rightarrow(P(x) Y \rightarrow P(x) Z)$

(8); Def. 7.

Por razones de espacio basten estos ejemplos como indicación de la riqueza de nuestro modelo $N_{1}{ }^{*}$. Dejamos para otra oportunidad: (a) su plena justificación filosófica y (b) los problemas técnicos, v.gr., como el de la decisión en $N_{1}{ }^{\circ}$.

Universidad de San Carlos, Guatemala.

Hector Neri Castañeda 